NASA Technical Memorandum 101962

\title{
Acidic Attack of Perfluorinated Alkyl Ether Lubricant Molecules by Metal Oxide Surfaces
}

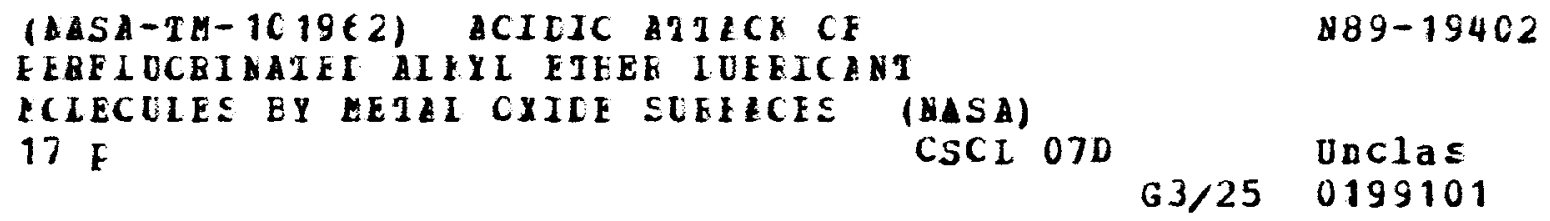

Michael J. Zehe and Owen D. Faut

Lewis Research Center

Cleveland, Ohio

Prepared for the

Joint Tribology Conference

cosponsored by the STLE and ASME

Fort Lauderdale, Florida, October 16-19, 1989 


\title{
ACIDIC ATTACK OF PERFLUORINATED ALKYL ETHER LUBRICANT
}

\author{
MOLECULES BY METAL OXIDE SURFACES \\ Michael J. Zehe and Owen D. Faut* \\ National Aeronautics and Space Administration \\ Lewis Research Center \\ Cleveland, Ohio 44135
}

\begin{abstract}
SUMMARY
The reactions of linear perfluoropolyalkylether (PFAE) lubricants with $\alpha-\mathrm{Fe}_{2} \mathrm{O}_{3}$ and $\mathrm{Fe}_{2} \mathrm{O}_{3}$-based solid superacids were studied. The reaction with $\alpha-\mathrm{Fe}_{2} \mathrm{O}_{3}$ proceeds in two stages. The first stage is an initial slow catalytic decomposition of the fluid. This reaction releases reactive gaseous products which attack the metal oxide and convert it to $\mathrm{FeF}_{3}$. The second stage is a more rapid decomposition of the fluid, effected by the surface FeF 3 . A study of the initial breakdown step was performed using $\alpha-\mathrm{Fe}_{2} \mathrm{O}_{3}, \alpha-\mathrm{Fe}_{2} \mathrm{O}_{3}$ preconverted to $\mathrm{FeF}_{3}$, and sulfate-promoted $\alpha-\mathrm{Fe}_{2} \mathrm{O}_{3}$ superacids. The results indicate that the breakdown reaction involves acidic attack at fluorine atoms on acetal carbons in the linear PFAE. Possible approaches to combat the problem are outlined.
\end{abstract}

\section{INTRODUCTION}

As newer aerospace systems are devised for operation at higher speeds and altitudes, their lubrication systems must perform in much more severe environments. The development of the next generation of advanced gas turbine engines may require a liquid lubricant capable of operation at temperatures in excess of $350^{\circ} \mathrm{C}$ in oxidizing environments (ref. 1). For a lubricant to be successful in the se operating conditions, it must not only possess a wide liquid range and good temperature-viscosity characteristics, but it must be thermally and oxidatively stable. Perfluoroalkylether (PFAE) compounds are good candidates for use in these high temperature applications (ref. 2). The inherent thermodynamic stability of the $\mathrm{C}-\mathrm{C}, \mathrm{C}-\mathrm{O}$, and $\mathrm{C}-\mathrm{F}$ bonds, and the chemically inert nature of their $\mathrm{C}-\mathrm{O}-\mathrm{C}$ ether linkage, make these molecules more stable to thermal and thermo-oxidative breakdown than comparable hydrocarbons. This thermal and chemical stability has led to the development in recent years of a number of PFAE fluids, with somewhat differing chemical structures. Their primary use has been as diffusion pump fluids for high vacuum pumping, but a number of these fluids are being considered for high temperature lubrication. The various fluids which have been developed are similar in many respects, e.g. they are all long chain polyethers comprised of short monomeric segments. Structural differences exist between the various fluid types, however, and these structural differences between the available PFAE's translate to large variabilities in their chemical stabilities at high temperature. It is felt that an examination of these reactivity differences can lead to an understanding of the mechanisms operative during thermal and thermo-oxidative breakdown. This knowledge can aid both in devising schemes to block the degradation and in development of newer, higher temperature compound classes. Possible schemes for improvement of the PFAE behavior at high temperature include changing the ir

*National Research Council - NASA Research Associate. 
molecular structures, the use of antioxidant additives, and modification of the (catalytic) surfaces which may promote their degradation.

In figures $1(a)$ and (b) are shown the structures of two PFAE fluids which are primary candidates for high temperature lubrication applications. The branched fluid shown in figure $1(a)$, consisting of repeating hexafluoroisopropoxy units, is produced by the anionic polymerization of hexafluoropropylene oxide. This material is thermally stable to temperatures above $400{ }^{\circ} \mathrm{C}$. In addition, it is resistant to attack by strong acids and bases at high temperatures (ref. 3). This thermal and chemical stability makes this fluid a strong candidate for high temperature lubrication applications. One undesirable property of this fluid which limits its use for high temperature lubrication, however, is that its viscosity versus temperature curve is too steep to allow its use over a wide enough temperature range for many applications ( $r e f$. 4). In figure $1(b)$ is shown the structure of a second PFAE fluid which has better temperature-viscosity characteristics (ref. 4), and may also have high temperature applicability. It is an unbranched fluid which is made by polymerizing tetrafluoroethylene in the presence of oxygen (ref. 5). The material is a random copolymer of difluoromethylene oxide and tetrafluoroethylene oxide. The thermal stability of this fluid is excellent, and its oxidation resistance is quite high in the absence of metal surfaces (ref. 6). The decomposition temperatures can be above $350^{\circ} \mathrm{C}$ under these conditions. In the presence of certain metals and alloys, however, oxidative decomposition is much more severe. The precise role of the metal in the degradation is not clear, but the catalytic breakdown requires the presence of oxygen (ref. 7). This catalyzed oxidative breakdown does not occur for the branched fluid. The difference in thermo-oxidative stability of the two fluids is paralleled by a corresponding difference in their reactivity towards Lewis acids. Whereas the branched fluid is stable to attack by strong acids and bases at temperatures above $350^{\circ} \mathrm{C}$ (ref. 3), the unbranched fluid suffers severe breakdown with the Lewis acid $\mathrm{AlCl}_{3}$ at temperatures as low as $100^{\circ} \mathrm{C}$ (ref. 8). It is possible that a common underlying reason may account for differences in both the chemical stability and the thermo-oxidative behavior of these two fluids.

It is worthwhile to consider in some detail the structural differences between the two fluids under discussion, and consider how these differences might relate to their chemical stabilities. As figure 1 shows, one difference between the branched and unbranched PFAE's is the presence on the former of a pendant perfluoromethyl group. It is possible that this group can sterically protect the oxygen atom towards attack and subsequent cleavage of the ether linkage. This protection of the ether group could account for the lower reactivity of the branched fluid towards acidic reagents. A second major difference between the two fluids is the presence in the unbranched fluid of acetal linkages, defined as the presence of an $0-C_{2}-0$ sequence in the chemical structure of the molecular chain. In hydrocarbon ethers, the presence of an acetal linkage greatly increases the reaction rate toward acid cleavage of the ether. It has been argued that the acetal linkage plays a major role in the oxidative degradation of PFAE fluids in the presence of water (ref. 9). This conclusion was reached by comparing the reactivity of two different PFAE structures, with and without acetal linkages. The reasons for the greater reactivity of acetal linkage-containing PFAE's are not entirely understood. Nonetheless, the greater reactivity of the unbranched fluid is of interest for understanding the degradation processes. 
In this paper, it is proposed that the initiation step of the oxidative decomposition of the unbranched PFAE is the partial transfer of electron density from unshared electron pairs on atoms in the linear PFAE molecule to Lewis acid sites at the surface of the (oxidized) metal surface. This initiation step is followed by the rapid unzipping of the molecules. This acidic attack by the metal oxide at basic sites on the PFAE is reasonable in light of what is known about the chemical stability of the unbranched PFAE. In addition to its instability towards Lewis acids as noted above, it decomposes when in contact with metal oxides such as $\mathrm{ZrO}_{2}$ and $\mathrm{Al}_{2} \mathrm{O}_{3}$ at elevated temperatures (refs. 9 and 10) and the presence of Lewis acid sites on the surface of many metal oxides is well documented (ref. 11). These acidic sites result from unfulfilled bonds and other surface defects which are electron-deficient. A reasonable mechanism for the initiation of fluoroether breakdown in the presence of oxygen and oxidizable metals is thus the formation of a surface metal oxide, whose acidic sites can react with the (basic) fluorine or oxygen atoms in the molecule. Partial electron transfer from the atom is followed by cleavage of adjacent bonds, starting an unzipping process which unravels the chain. The observed large variation in reactivity from oxide to oxide is dependent upon the density of acidic sites on the surface and their degree of acidity; it is well known that these two parameters vary considerably from oxide to oxide (ref. 11). The experiments described in this report were undertaken to test the hypothesis that reactivity is directly related to both the density and degree of acidity of surface acid sites.

In these experiments, the rate of reaction was studied for a commercial PFAE fluid in contact with powdered $\alpha-\mathrm{Fe}_{2} \mathrm{O}_{3}$. The reactions were performed under dynamic vacuum, and the onset of fluid decomposition was indicated by a pressure $r i s e$ in the system. In addition to the experiments involving $\alpha-\mathrm{Fe}_{2} \mathrm{O}_{3}$, a number of experiments were performed in which the surface was treated to enhance the surface acidity. These involved the treatment of the oxide with $\mathrm{COF}_{2}$, and the use of $\mathrm{Fe}_{2} \mathrm{O}_{3}$-based superacids.

\section{EXPERIMENT}

\section{Materials}

The structure of the unbranched PFAE fluid used in this research is shown in figure 1(a). Its average molecular weight is 10000 , and the ratio of perfluoromethyl ether to perfluoroethyl ether units is 0.6:0.7. The branched PFAE fluid structure is shown in figure $1(b)$.

$\alpha-\mathrm{Fe}_{2} \mathrm{O}_{3}$ was synthesized using standard inorganic synthetic routes (ref. 12). Reagent grade $\mathrm{Fe}\left(\mathrm{NO}_{3}\right)_{3} \cdot 9 \mathrm{H}_{2} \mathrm{O}$ was dissolved in low conductivity deionized water and dried to $\mathrm{Fe}(\mathrm{OH})_{3}$. The $\mathrm{Fe}(\mathrm{OH})_{3}$ was heated at $300{ }^{\circ} \mathrm{C}$ for 3 to $4 \mathrm{hr}$ to convert it to $\alpha-\mathrm{Fe}_{2} \mathrm{O}_{3}$. X-ray diffraction of the product showed lines due to $\alpha-\mathrm{Fe}_{2} \mathrm{O}_{3}$ only. X-ray photoelectron spectroscopy (XPS) showed only $\mathrm{Fe}$ and $\mathrm{O}$ peaks. Sulfate-activated solid $\mathrm{Fe}_{2} \mathrm{O}_{3}$ superacids were synthesized according to the procedure of Yamaguchi et al. (ref. 13); XPS spectra showed only $\mathrm{Fe}, 0$, and $\mathrm{S}$ peaks. $\mathrm{COF}_{2}$ gas was used as received. Mass spectrometry showed minor ( $<$ percent) impurity peaks attributable to $\mathrm{CF}_{3} \mathrm{COF}$ and $\left(\mathrm{CF}_{3}\right)_{2} \mathrm{CO}$. 


\section{FLUID DECOMPOSITION TEST APPARATUS AND PROCEDURE}

The apparatus used to determine stability of the fluid is shown in figure 2. A $5 \mathrm{ml}$ borosilicate glass bulb containing the fluid, powdered oxide, and a polytetrafluoroethylene-coated stirring bar, was attached to a vacuum manifold through a stopcock. A thermocouple gauge tube was used to monitor the pressure above the fluid. The entire test procedure, including degassing and reaction, was performed under active pumping conditions. The reactor was evacuated by a commercial liquid nitrogen-cooled sorption pump. The glass bulb was heated by immersing it in a hot oil bath whose temperature was measured with a chromel-alumel thermocouple. A magnetic stirring bar in the hot oil bath coupled with the stirring bar in the reaction bulb to agitate the powder/lubricant mix and ensure intimate contact between the powder and the liquid. In the procedure, the bulb was first charged with 50 to $60 \mathrm{mg}$ powdered oxide and $\approx 1.5 \mathrm{~g}$ lubricant. The bulb was evacuated to 10 mtorr or less, and was then degassed at $150^{\circ} \mathrm{C}$. During degassing, the pressure rose to 50 to 100 mtorr. Degassing was judged complete when the pressure fell again below 10 mtorr. The bulb was then removed from the oil bath, and the bath was heated to the desired reaction temperature. The reaction bulb was then reimmersed in the bath. The pressure as a function of time was recorded. The reaction time of the powder/lubricant mix was defined as the time it took for the pressure to reach 200 mtorr. This reaction time will henceforth be termed the "induction period."

\section{MASS SPECTROMETER EXPERIMENTS}

In addition to the decomposition tests described above, a number of experiments were performed in which the gaseous decomposition products were analyzed with a mass spectrometer. For these studies, a triple quadrupole mass spectrometer was used in the single mass spectrometer mode. The gaseous decomposition products were routed to the ionizer through a glass U-trap held at 0 or $-78^{\circ} \mathrm{C}$, depending on the objective of the experiment. Mass spectra were obtained in the electron impact mode using $70 \mathrm{eV}$ electron energy. The instrument was calibrated for mass location and intensity with the standard calibration compound perfluorotributylamine (FC 43).

\section{CHEMICAL ANALYSIS}

Surface fluoride analysis of one batch of reacted powder was performed using wet chemical techniques. The lubricant was first dissolved and removed from the powder with trichlorotrifluoroethane under dry nitrogen. A small quantity of deionized water was then added to the powder to dissolve the surface fluoride. Next, the slurry was filtered to remove the powder, and the fluoride concentration in the clear liquid was measured with an electrode specific for fluoride. The liquid was then tested for dissolved iron using atomic absorption spectroscopy. A blank was run in which a quantity of unreacted powder was slurried with the unbranched PFAE, but never heated. The powder from the unreacted slurry was then extracted and treated in an identical manner as the sample had been. 


\section{RESULTS}

\section{Fluid Decomposition}

Figure 3 shows the pressure-time plot which is obtained when $1.5 \mathrm{~g}$ of the unbranched PFAE fluid is heated with $50 \mathrm{mg} \alpha-\mathrm{Fe}_{2} \mathrm{O}_{3}$ at $\mathrm{T}=185^{\circ} \mathrm{C}$. The shape of the curve is typical of the reaction: first, there is an initial short period in which there is no apparent reaction, followed by a gradually accelerating pressure rise until the pressure exceeds the gauge's upper limit of 1 torr. At this point, the lubricant/powder mix is observed to be bubbling vigorously up the stem of the reaction vessel. Maintaining the temperature at $185^{\circ} \mathrm{C}$ results in complete loss of the fluid in 15 to $20 \mathrm{~min}$. Quenching the vessel to room temperature results in an immediate cessation of the reaction, and reheating it causes the reaction to resume immediately. The induction period which was measured for the decomposition of the unbranched fluid in contact with pure $\alpha-\mathrm{Fe}_{2} \mathrm{O}_{3}$ was $8.0 \mathrm{~min}(\sigma=1.6 \mathrm{~min})$.

When the branched fluid was heated in contact with the powder at $185{ }^{\circ} \mathrm{C}$, no pressure rise occurred and no physical changes in the fluid/powder mix could be detected during a $1 \mathrm{hr}$ period, except for a brief initial pressure rise upon heating due to release of dissolved gases. Heating the $\mathrm{mix}$ to $205^{\circ} \mathrm{C}$ for $1 \mathrm{hr}$ also resulted in no changes.

An experiment was performed to determine if the reaction between the unbranched fluid and the $a-\mathrm{Fe}_{2} \mathrm{O}_{3}$ involved a change in the powder, the lubricant, or both. The reaction was quenched after the pressure had reached 1 torr, and the bulb was removed from the vacuum line and centrifuged to separate the powder from the liquid. The lubricant was removed from the bulb, a fresh charge of liquid was added to the powder, and the mixture was degassed. Heating to $185^{\circ} \mathrm{C}$ resulted in a reaction which was almost immediate; the induction period was $40 \mathrm{sec}$. In contrast to this behavior, when the fluid which had been removed from the reaction vessel was added to fresh $\alpha-\mathrm{Fe}_{2} \mathrm{O}_{3}$, degassed, and heated to $185^{\circ} \mathrm{C}$, the measured induction period was $8 \mathrm{~min}$. This result indicates that a fundamental change had occurred in the powder, but not the fluid, during the reaction.

The wet chemical test described above was used to analyze the powders for fluoride and soluble iron. A small quantity $(0.15 \mathrm{~g})$ of reacted $\mathrm{Fe}_{2} \mathrm{O}_{3}$ was extracted and slurried in $15 \mathrm{ml}$ deionized $\mathrm{H}_{2} \mathrm{O}$. This treatment will dissolve soluble fluorides such as $\mathrm{FeF}_{3}$, but not the unreacted oxide. The fluoride ion concentration in the liquid was measured to be $0.4 \mu \mathrm{mol} / \mathrm{liter}$ (blank = $0.02 \mu \mathrm{mol} / \mathrm{liter}$ ). The iron concentration was $0.2 \mu \mathrm{mol} / \mathrm{i}$ iter (blank = $0.006 \mu \mathrm{mol} / \mathrm{liter}$ ).

\section{MASS SPECTROMETER ANALYSIS}

Figure 4 shows a mass spectrum of the volatile vapors evolved from the reaction vessel during the "vigorous reaction" stage of the experiment. To remove any carryover lubricant, the vapors were routed through a cold trap held at $-78{ }^{\circ} \mathrm{C}$ enroute to the mass spectrometer. The mass spectrum shows strong peaks due to $\mathrm{CO}_{2}(44,28)$, and $\mathrm{COF}_{2}(28,47,66)$. In addition, there are weaker peaks assignable to $\mathrm{CF}_{3} \mathrm{CFO}(69,116)$. The extensive fragmentation which occurs in the electron impact spectrum of fluoroethers and fluoracids makes mass spectrometry of limited value in determining the structures of all 
the volatile components evolved, and attempts to minimize fragmentation (lower electron energy, chemical ionization techniques) were unsuccessful. Nonetheless, the presence of $\mathrm{COF}_{2}$ is definite and $\mathrm{CF}_{3} \mathrm{CFO}$ very probable, as volatile decomposition products which are generated at the powder-liquid interface.

Figure 5 shows the results of an experiment in which the evolution of $\mathrm{CO}_{2}$ and $\mathrm{COF}_{2}$ were continuously monitored during the early stages of the experiment, before vigorous bubbling had commenced. It can be seen that the relative amounts of evolved $\mathrm{CO}_{2}$ and $\mathrm{COF}_{2}$ change dramatically during the early stages of the reaction. Initially, the evolved gases are more concentrated in $\mathrm{CO}_{2}$, but gradually the $\mathrm{COF}_{2}$ content rises, and the $\mathrm{CO}_{2}$ content decreases.

The changing $\mathrm{COF}_{2} / \mathrm{CO}_{2}$ ratio in the early products, and the presence of $\mathrm{F}^{-}$ on the surface of the powder, suggested that the following reaction was taking place:

$$
\mathrm{Fe}_{2} \mathrm{O}_{3}(\mathrm{~s})+3 \mathrm{COF}_{2}(\mathrm{~g})=2 \mathrm{FeF}_{3}(\mathrm{~s})+3 \mathrm{CO}_{2}(\mathrm{~g})
$$

For this reaction, the standard free energy change, $\Delta G^{\circ}$, is calculated to be $-123 \mathrm{kcal} / \mathrm{mol}$ at $500{ }^{\circ} \mathrm{K}$ ( $\mathrm{ref}$. 14). A slow conversion of the $\mathrm{Fe}_{2} \mathrm{O}_{3}$ to $\mathrm{FeF}_{3} \mathrm{via}$ this reaction can qualitatively explain the change in the $\mathrm{CO}_{2} / \mathrm{COF}_{2}$ ratio during the early stages of the run. The initial slow decomposition of the fluid, slowly catalyzed by $\mathrm{Fe}_{2} \mathrm{O}_{3}$, releases both $\mathrm{CO}_{2}$ and $\mathrm{COF}_{2}$ at the solid liquid interface. Much of the $\mathrm{COF}_{2}$ which initially forms is used up in reaction ( 1 ), resulting in a relatively higher $\mathrm{CO}_{2} / \mathrm{COF}_{2}$ ratio. Eventually, the surface of the oxide is completely converted to the fluoride. At this point, $\mathrm{COF}_{2}$ is no longer used in reaction (1), and it is released as a gas. The $\mathrm{CO}_{2} / \mathrm{COF}_{2}$ ratio thus falls to its "normal" value as determined by the decomposition of the fluid.

\section{COF$_{2}$ PRETREATMENT}

To test this hypothesis, one experiment was performed in which the powder/ liquid mix was first degassed at $150^{\circ} \mathrm{C}$ in vacuo, and was then treated at $110{ }^{\circ} \mathrm{C}$ with a small quality ( 50 standard $\mathrm{ml}$ ) COF 2 through an immersed bubbler tube. When the resultant powder/lubricant mix was heated to $185^{\circ} \mathrm{C}$, reaction was almost immediate: time to 200 mtorr was $50 \mathrm{sec}$. This compares with the result of $8 \mathrm{~min}$ for the untreated powder. This dramatic lowering of the induction period with $\mathrm{COF}_{2}$ treatment of the reactants supports the hypothes is that

$\mathrm{COF}_{2}$ is indeed converting the surface to $\mathrm{FeF}_{3}$, which, because of its high acidity, is more effective in promoting the fluid degradation.

\section{SUPERACID EXPERIMENTS}

To further test the reactivity of the linear PFAE fluid towards acidic surfaces, its behavior was studied in the presence of a powdered solid superacid, namely, $\mathrm{Fe}_{2} \mathrm{O}_{3}$ promoted with sulfate. This material consists of a solid matrix of $\alpha-\mathrm{Fe}_{2} \mathrm{O}_{3}$ with a small amount of sulfur oxide on its surface. The sulfur-oxygen atomic arrangement is a unique bridging structure (ref. 13) which lends very high acidity to the surface, with Hammett $\left(H_{0}\right)$ values $<-12.5$ (ref. 15). This means that the acidity of the material, as measured by $\mathrm{H}_{0}$, is 100 times that of concentrated $\mathrm{H}_{2} \mathrm{SO}_{4}$. The exceedingly high Lewis acidity manifests itself in a very high catalytic activity in acid-catalyzed chemical 
reactions; e.g., the rate of catalytic dehydration of 2-butanol is enhanced two hundredfold over unpromoted $\alpha-\mathrm{Fe}_{2} \mathrm{O}_{3}$. XPS and IR spectroscopies have shown that the surface acidity is related to the presence of $\mathrm{S}^{+6}$; further, catalytic activity towards hydrocarbon reactions can be removed (ref. 13) by $\mathrm{H}_{2}$ reduction at $350{ }^{\circ} \mathrm{C}$ with reduction of $\mathrm{S}^{+6}$ to $\mathrm{S}^{-2}$. Because of the ability of this material to enhance acidic reactions, it was decided to test its activity towards the decomposition of the linear PFAE fluid. Accordingly, a quantity of the material containing 2 percent $S$ was synthesized according to the method of Yamaguchi et al. (ref. 13). In figure $6(2)$ is shown the XPS spectrum of the material as synthesized; the presence of $s^{+6}$ is demonstrated by the presence of a peak at $170 \mathrm{eV}$ (ref. 13). Heating the material at $450{ }^{\circ} \mathrm{C}$ in 1 torr $\mathrm{H}_{2}$ yields material whose XPS spectrum is given in figure $6(b)$. Partial reduction of $S^{+6}$ to $\mathrm{S}^{-2}$ is apparent in the appearance of a second peak at a binding energy $7 \mathrm{eV}$ lower. Thus, the surface analysis of the synthesized material indicates that it is indeed the desired $\mathrm{Fe}_{2} \mathrm{O}_{3}$-based superacid.

When the superacid was tested as a catalyst for the PFAE decomposition, the reaction was very rapid, with a measured induction period of $1 \mathrm{~min}$ ( $\sigma=$ $30 \mathrm{sec}$ ). This compares with the induction period of $\mathrm{COF}_{2}$-treated $\mathrm{Fe}_{2} \mathrm{O}_{3}$, also $\approx 1 \mathrm{~min}$, and the much longer value of $8 \mathrm{~min}$ for $\alpha-\mathrm{Fe}_{2} \mathrm{O}_{3}$.

\section{DISCUSSION}

The results of these experiments lend strong support to the hypothesis that the oxidative breakdown of linear PFAE fluids in the presence of oxidizable metals is a reaction between acidic sites on the surface of the partially oxidized metal, and basic sites on the molecular chain. Further, the reaction is autocatalytic: the initial slow breakdown by weakly acidic sites on the $\mathrm{Fe}_{2} \mathrm{O}_{3}$ results in gaseous $\mathrm{COF}_{2}$ and other reactive products, which convert the surface to $\mathrm{FeF}_{3}$. The resultant surface is highly effective for further breakdown because it is strongly acidic and because the density of acidic sites is very high. The acidic surface can serve as an effective receptor for available electrons from the PFAE. Polarization and distortion of the PFAE molecule can allow cleavage of an adjacent bond, and unzipping of the chain. It is postulated that the initial step, the acidic attack of the polymer, is rate-determining.

To more fully understand the initiation step, one must identify the site of the attack on the chain. There are two sites on the chain which are vulnerable to this attack: the electron-rich fluorine atoms, and the lone-pair electrons on the oxygen atoms. Considering first the oxygen atoms, one notes that for hydrocarbon ethers a characteristic reaction is cleavage of the ether linkage, initiated by acidic attack of the oxygen atom. The reaction sequence is shown in figure $7(a)$. Of particular interest is the role played by the presence of an acetal linkage. In hydrocarbon ethers, the presence of this $0-C-0$ sequence increases the reaction rate by reducing the energy of the transition state, because of the strong contribution of the structures shown in figure $7(\mathrm{c})$. The extra stabilization which these structures give the carbenium ion makes the reaction leading to it much more favorable than those which result from nonacetal ether bridges.

One must next consider the differences in reactivity and reaction mechanisms which result when fluorine atoms replace hydrogen in organic systems. As the most electronegative element, fluorine has a major effect on $\alpha$-atom 
basicity when it substitutes for hydrogen. Thus, for example, trimethylamine,

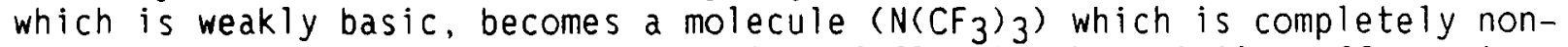
basic (ref. 17). The electronegativity of fluorine has similar effects in those aliphatic ethers which have been studied; e.g., simple perfluoroalkyl ethers are stable to $H I$ cleavage, and do not complex with BF 3 (ref. 18). The effect of the fluorine atoms in the PFAE molecules should be similar, i.e., stabilization of the ether to acid attack at the oxygen atoms. This would suggest that for the PFAE's, electrophilic attack by Lewis acids should occur not at the oxygen atoms, but elsewhere on the chain.

A second possibility for the site of attack on the PFAE chains are the electron-rich fluorine atoms. This initiation step has no analog in hydrocarbon chemistry, but a number of studies have shown that in favorable environments, these nonbonding electrons are available for transfer to acidic reactants (refs. 19 and 20). These favorable conditions are provided by the $0-\mathrm{CF}_{2}-\mathrm{O}$ sequence in the acetal linkage. Abstraction of either fluorine atom results in a carbenium ion which is especially stable, because it is resonancestabilized by two adjacent oxygens atoms, and because there are no $\alpha$-fluoromethylene groups, which are known to destabilize the carbenium ion (ref. 21). For these reasons, therefore, it is felt that the reactivity of the particular unbranched PFAE structure under consideration, towards Lewis acids, $\mathrm{Fe}_{2} \mathrm{O}_{3}$, $\mathrm{FeF}_{3}$, and sulfate-based superacids, involves acid attack and abstraction of acetal fluorine atoms, rather than attack of the ether oxygen atoms. The importance of the acetal linkage was established by the absence of reaction for the branched fluid; the lack of basic character of the oxygen atoms means that the reaction is initiated elsewhere on the molecule. The autocatalytic behavior of the system is a result of the conversion of the surface to highly catalytic $\mathrm{FeF}_{3}$, during early stages of the process.

\section{CONCLUSIONS}

The presence of metal oxide surfaces in contact with acetal-containing PFAE lubricants will inevitably result in acidic breakdown of the ethers.

There is little which can be done to the PFAE chains to block the acidic attack without compromising the viscosity-temperature qualities which result from perfluoromethylene groups, and it is the presence of these groups which leads to the acetal structure. This means that solutions to the thermo-oxidative breakdown problem should focus on the surfaces which are in contact with the liquid. Some success in blocking thermo-oxidative degradation of linear PFAE's below $300{ }^{\circ} \mathrm{C}$ has been achieved with the use of additives containing nitrogen or phosphorous atoms (ref. 7). The action of these additives may depend upon their ability to complex acidic sites on the surface. Other approaches may involve surface modification to limit their reactivity towards the reactive gases which result from the initial decomposition, since it is the catalytic action of the resulting surface which accelerates the breakdown. The results which have been reported here suggest that an important factor in the choice of materials should be the surface acidity. Followup research to prove or disprove the hypotheses set forth in this report and to block the reactions observed should focus on (1) the detailed nature of the interaction between the acidic reagent and model PFAE's, contrasting acetal-containing molecules with those lacking this structure, (2) modification of the surfaces to assure that acidic sites do not form, and (3) additives to quench the carbenium intermediates which form or to complex the acidic surface. 


\section{REFERENCES}

1. P. Sutor, and W. Bryzik, "Development of Advanced High-Temperature Liquid Lubricants," Recent Developments in the Adiabatic Engine, SAE SP-738, Society of Automotive Engineers, Warrendale, PA, 1988, pp. 37-49.

2. C.E. Snyder, and R.E. Dolle, Jr., "Development of Polyperfluoroalkylethers as High Temperature Lubricants and Hydraulic Fluids," ASLE Transactions, Vol. 19, No. 3, July 1976, pp. 171-180.

3. W.H. Gumprecht, "PR-143 - A New Class of High-Temperature Fluids," ASLE Transactions, Vol. 9, No. 1, Jan. 1966, pp. 24-30.

4. C.E. Snyder, Jr., L.J Gschwender, and C. Tamborski, "Linear Polyperfluoroalkylether-Based Wide-Liquid-Range High-Temperature Fluids and Lubricants," Lubrication Engineering, Vol. 37, No. 6, June 1981, pp. 344-349.

5. D. Sianesi, and A. Pasetti, "Fluorinated Polyether Copolymers and Method for Preparing Them from Tetrafluoroethylene," U.S. Patent 3,715,378, Feb. $6,1973$.

6. K.L. Paciorek, S.R. Masuda, J.H. Nakahara, and R.H. Kratzer, "Improved Perfluoroalkylether Fluid Development," NASA CR-180872, 1987.

7. W.R. Jones, Jr., K.J.L. Paciorek, T.I. Ito, and R.H. Kratzer, "Thermal Oxidative Degradaticn Reactions of Linear Perfluoroalkyl Ethers," Industrial and Engineering Chemistry Product Research and Development, Vol. 22, No. 2, June 1983, pp. 166-170.

8. T.R. Bierschenk, H. Kawa, T.J. Juhlke, and R.J. Lagow, "The Development of New, Low-Cost Perfluoroalkylether Fluids with Excellent Low and High-Temperature Properties," NASA CR-182155, 1988.

9. W. Morales, "Surface Catalytic Degradation Study of Two Linear Perfiuoropolyalkylethers at $345^{\circ} \mathrm{C}, "$ NASA TP-2774, 1987.

10. D. Sianesi, V. Zamboni, R. Fontanelli, and M. Binaghi, "Perfluoropolyethers: Their Physical Properties and Behaviour at High and Low Temperatures," Wear, Vol. 18, 1971, pp. 85-100.

11. K. Tanabe, Solid Acids and Bases; Their Catalytic Properties, Academic Press, New York, 1970.

12. G. Brauer, ed., Handbook of Preparative Inorganic Chemistry, Second Edition, Academic Press, New York, 1963.

13. T. Yamaguchi, T. Jin, and K. Tanabe, "Structure of Acid Sites on SulfurPromoted Iron Oxide," Journal of Physical Chemistry, Vol. 90, No. 14, July 3, 1986, pp. 3148-3152.

14. JANAF Thermochemical Tables, Third Edition, American Chemical Society, Washington, D.C., 1986. 
15. M. Hino, and K. Arata, "Synthesis of Solid Superacid Catalyst with Acid Strength of $\mathrm{H}_{0} \leq-16.04$," Journal of the Chemical Society, Chemical Communications, No. 18, 1980, pp. 851-852.

16. G.A. Olah, Friedel-Crafts Chemistry, John Wi ley and Sons, Inc., New York, 1973.

17. F.A. Cotton, and G. Wilkinson, Advanced Inorganic Chemistry, A Comprehensive Text, Third Edition, John Wiley and Sons, Inc., New York, 1972,

18. R.E. Banks, Fluorocarbons and Their Derivatives, MacDonald \& Co. Ltd., London, 1970 .

19. G.A. Olah, J.R. DeMember, and R.H. Schlosberg, "Friedel-Crafts Chemistry. III. Methyl Fluoride-Antimony Pentafluoride, a Powerful New Methylating Agent. Methylation Reactions and the Polycondensation of Methyl Fluoride," Journal of the American Chemical Society, Vol. 91, No. 8, Apr. 9, 1969, pp. 2112-2113.

20. W.G. Liauw, and A.G. Harrison, "Site of Protonation in the Reaction of Gaseous Bronsted Acids with Halobenzene Derivatives," Organic Mass Spectrometry, Vol. 16, No. 9, 1981, pp. 388-392.

21. R.D. Chambers, Fluorine in Organic Chemistry, John Wiley and Sons, Inc., New York, 1973.

$$
\begin{gathered}
\mathrm{CF}_{3}-0-\left(\mathrm{CF}_{2}{ }^{0}\right)_{\mathrm{q}}-\left(\mathrm{CF}_{2} \mathrm{CF}_{2}{ }^{0}\right)_{\mathrm{p}}-\mathrm{CF}_{3} \\
\mathrm{p} / \mathrm{q}=(0.6-0.7)
\end{gathered}
$$

FIGURE 1A. - STRUCTURE OF LINEAR PFAE USED IN

THIS RESEARCH.

$$
\begin{gathered}
\mathrm{CF}_{3}-\left.\right|_{\left(\mathrm{CFCF}_{2}{ }^{0}\right)_{n}} ^{\mathrm{CF}_{3}}-\mathrm{CF}_{3} \\
\mathrm{n}=10-20
\end{gathered}
$$

FIGURE 1B. - STRUCTURE OF BRANCHED PFAE USED IN THIS RESEARCH. 


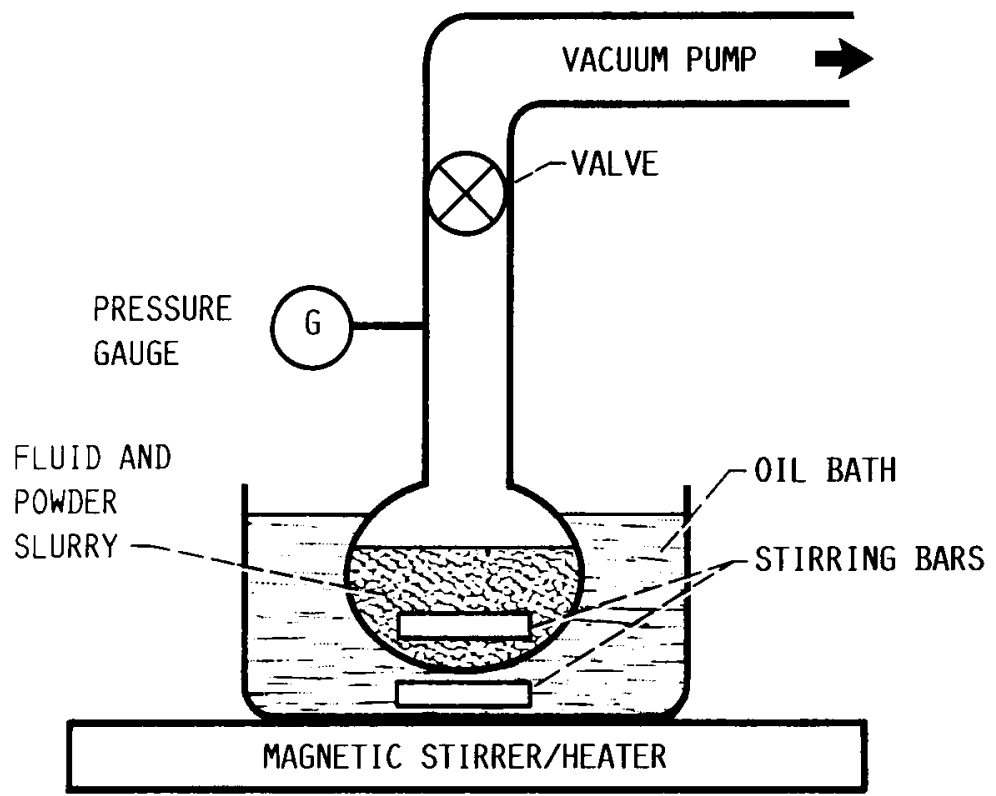

FIGURE 2. - EXPERIMENTAL APPARATUS FOR STUDYING THERMAL STABILITY OF PFAE FLUIDS.

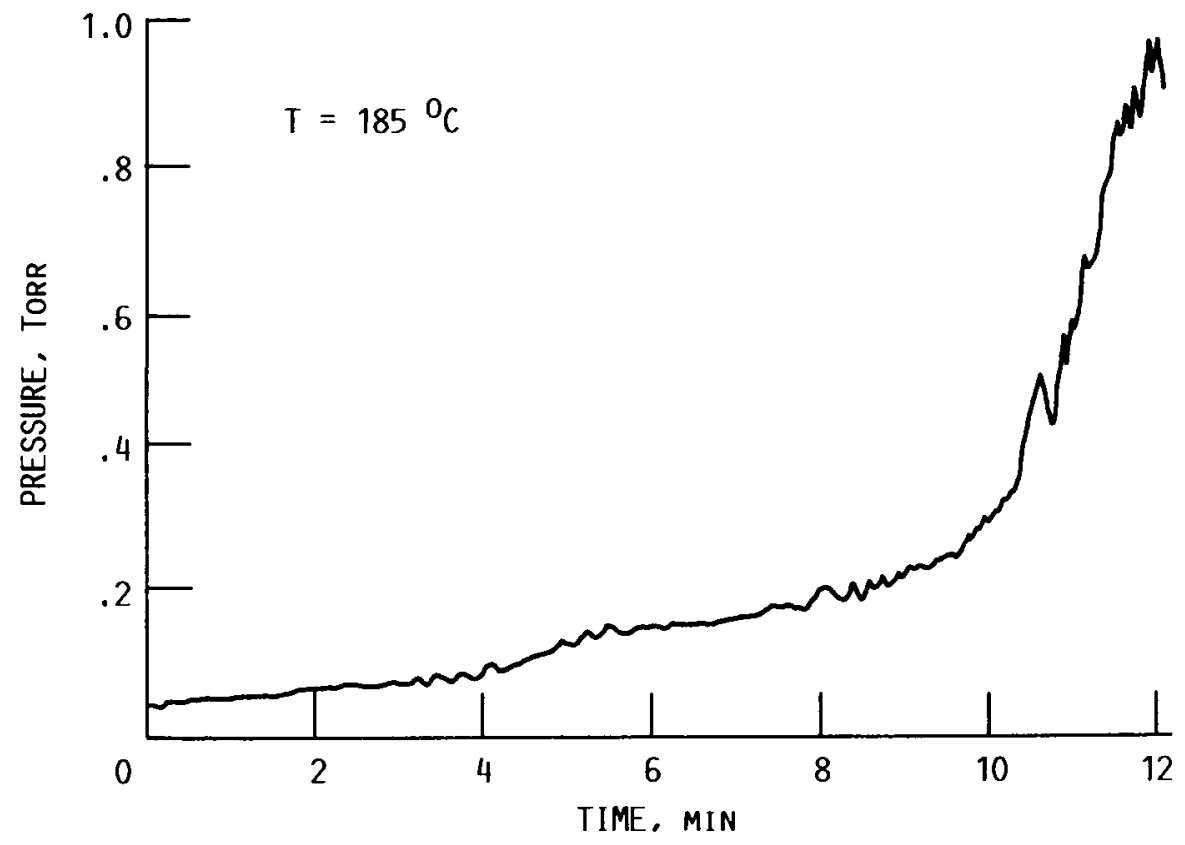

FIGURE 3. - PRESSURE-TIME RELATIONSHIP FOR DECOMPOSITION OF LINEAR PFAE IN CONTACT WITH $\alpha-\mathrm{Fe}_{2} \mathrm{O}_{3}$ AT $185^{\circ} \mathrm{C}$. 


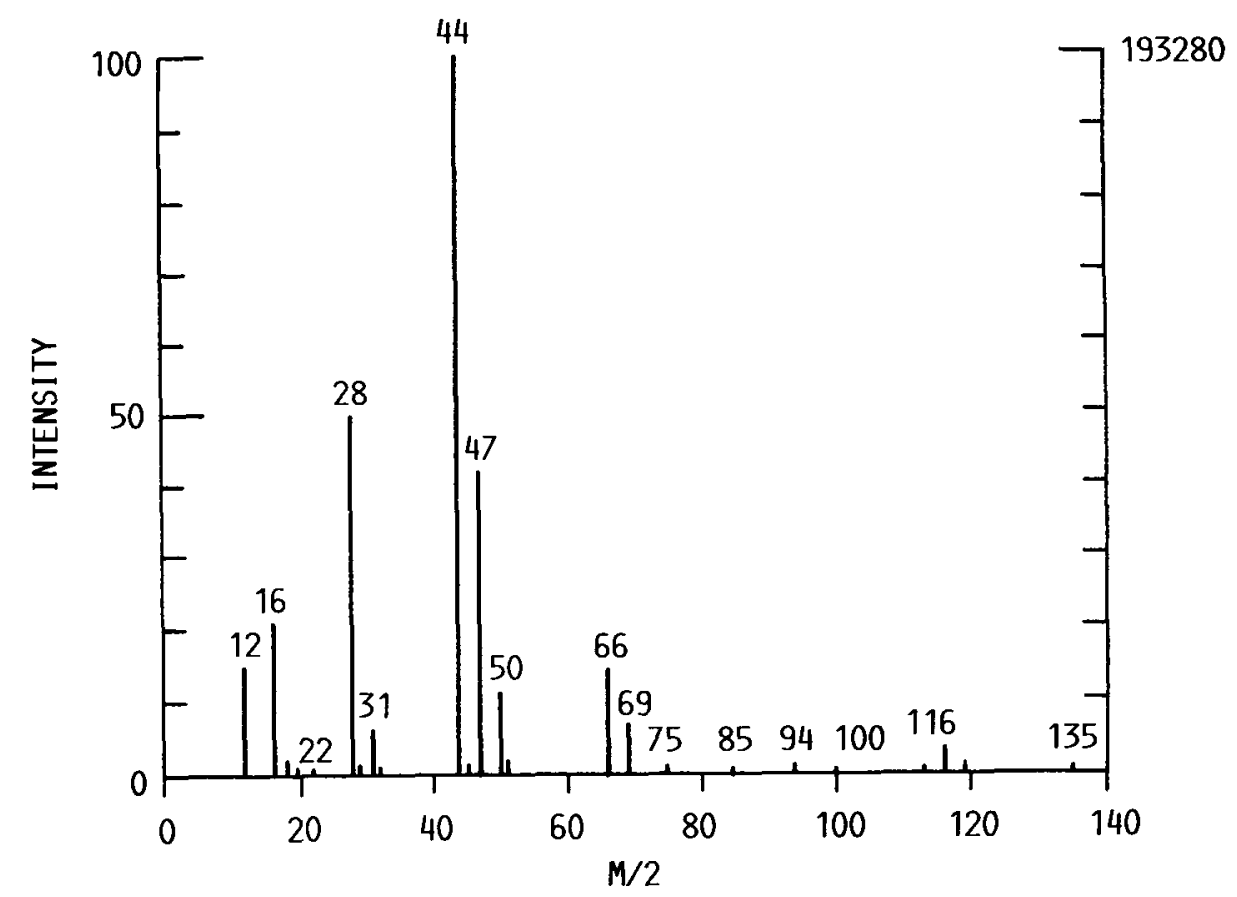

FIGURE 4. - MASS SPECTRUM OF VOLATILE VAPORS EVOLVED

FROM DECOMPOSITION OF LINEAR PFAE IN CONTACT WITH $a-\mathrm{FeAl}_{2} \mathrm{O}_{3}$ AT $185^{\circ} \mathrm{C}$. 


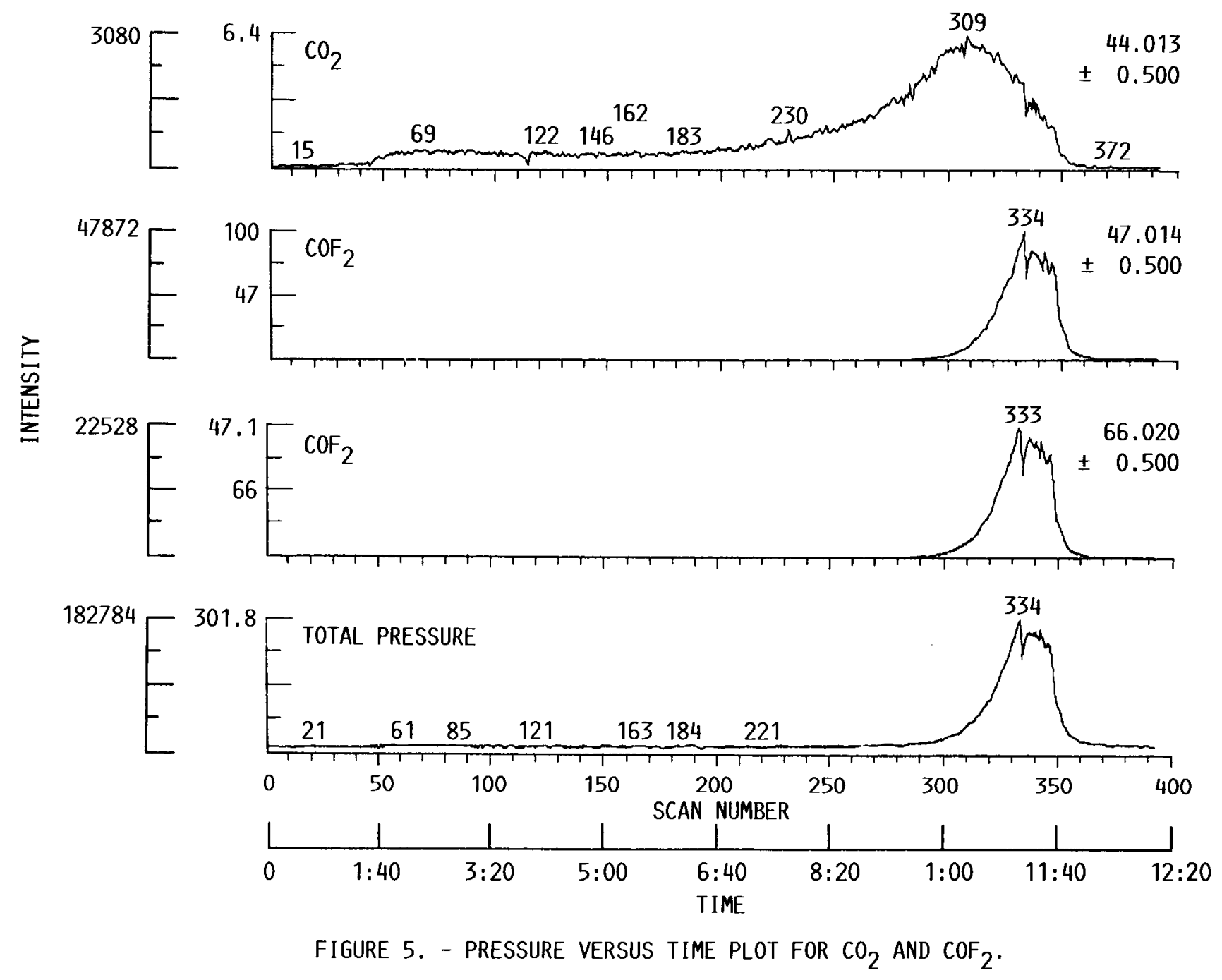




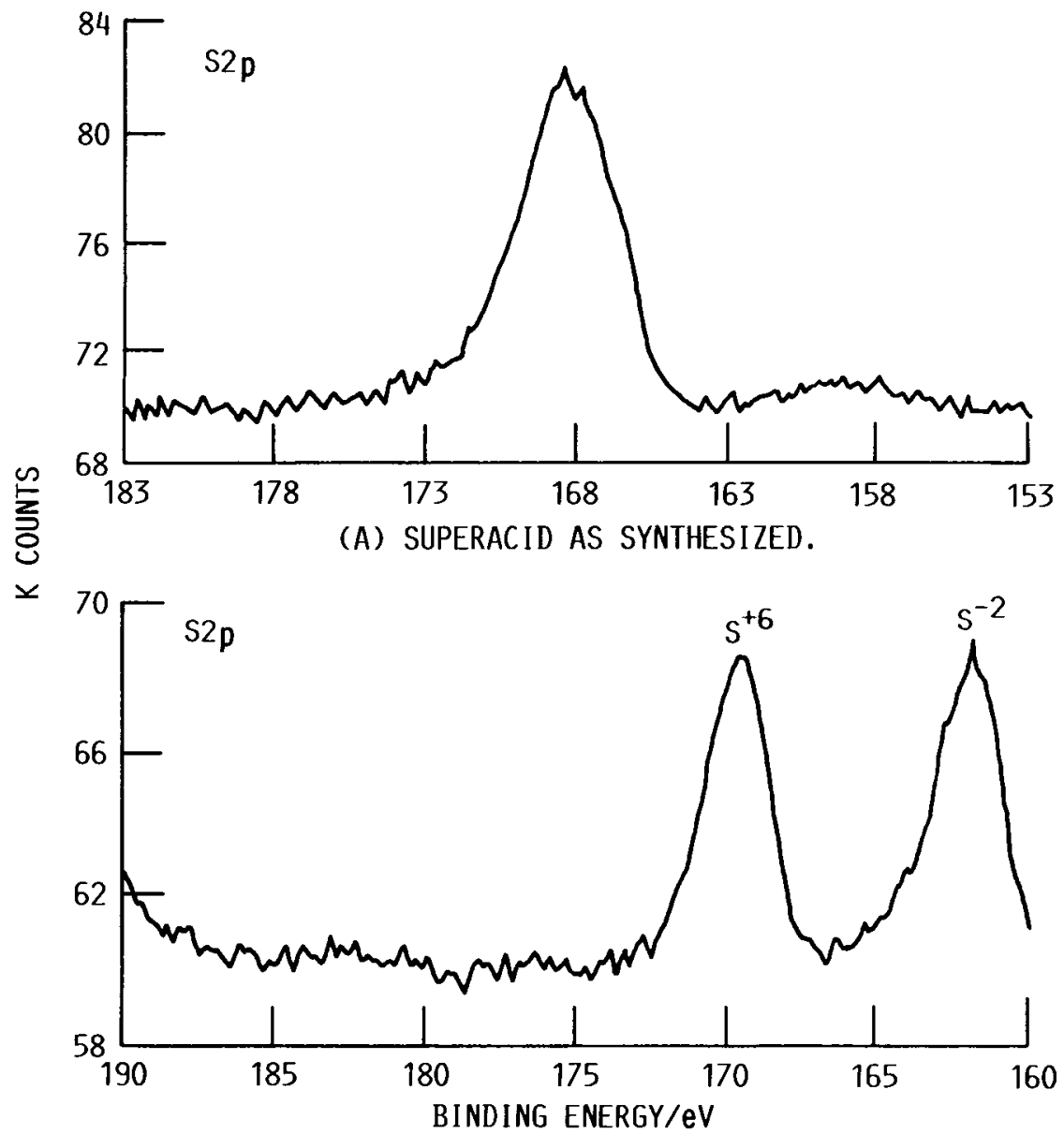

(B) BASED SUPERACID AFTER REDUCTION IN $\mathrm{H}_{2}$ AT $450{ }^{\circ} \mathrm{C}$.

FIGURE 6. - SULFUR 2p XPS SPECTRUM OF SULFATE-PROMOTED $\mathrm{Fe}_{2} \mathrm{O}_{3}$ 


$$
\begin{aligned}
& \mathrm{R}-\stackrel{\mathrm{H}}{\mathrm{C}}-\mathrm{O}-\mathrm{H}-\mathrm{C}-\mathrm{R}+\mathrm{A}^{+} \rightarrow \\
& \mathrm{H} H
\end{aligned}
$$

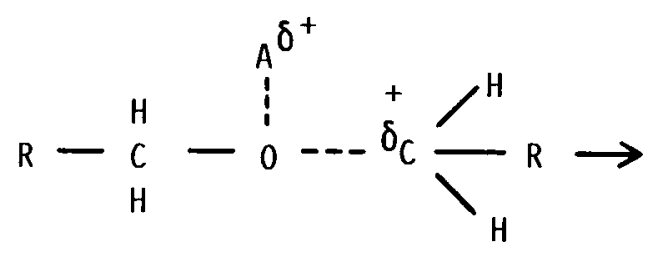

$$
\begin{aligned}
& \mathrm{R}-\mathrm{CH}_{2}-\mathrm{O}-\mathrm{A}+\oplus \mathrm{CH}_{2}--\mathrm{R}
\end{aligned}
$$

FIGURE 7A. - ACID-INITIATED ETHER CLEAVAGE.

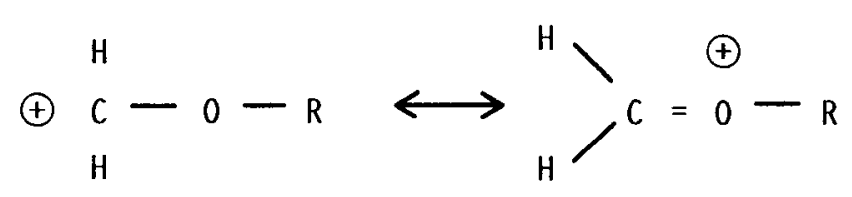

FIGURE 7B. - RESONANCE STABILIZATION OF ACETAL CARBENIUM ION. 


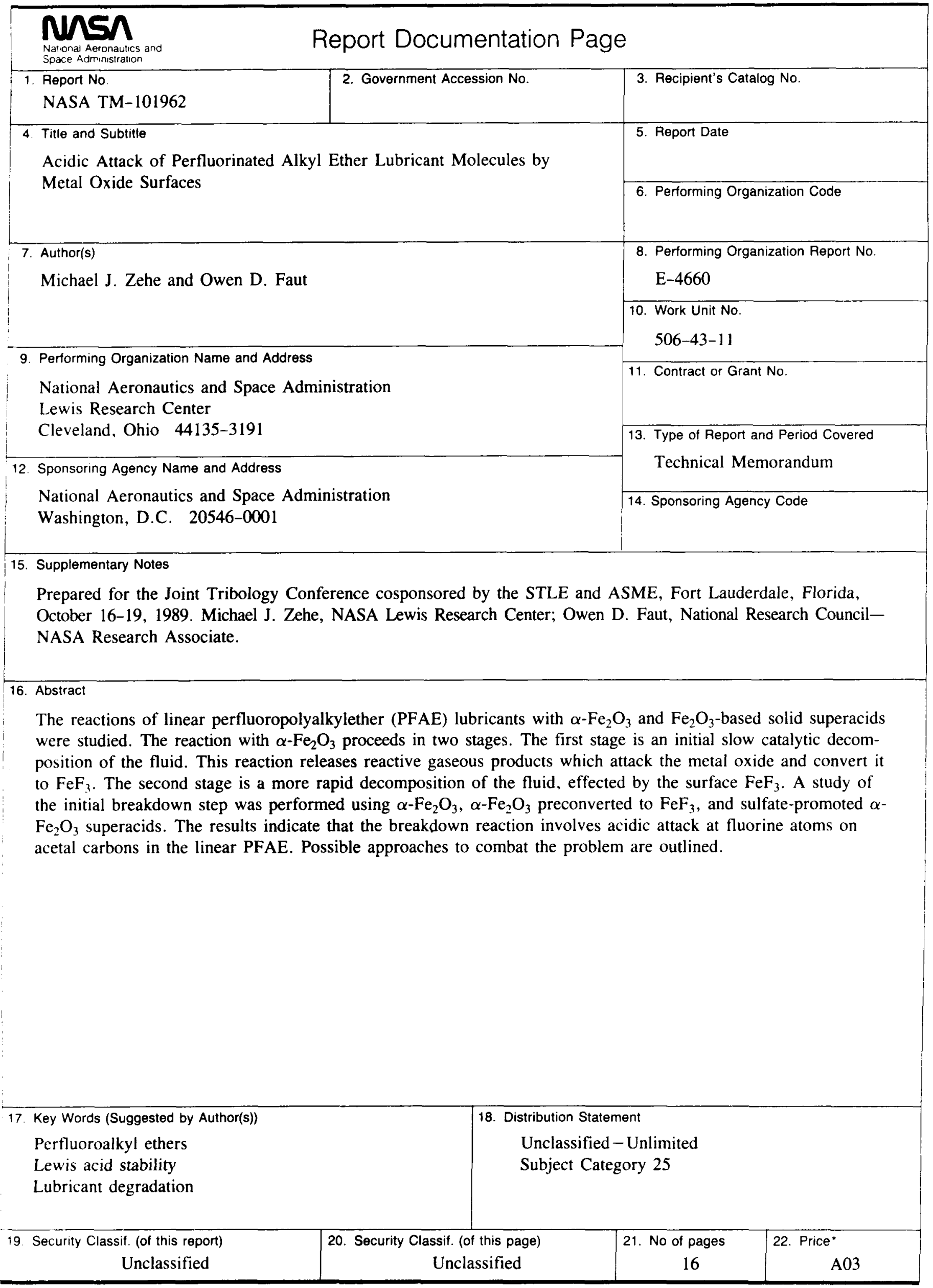

